\title{
Cooperative Coverage Extension in Land Mobile Satellite Networks
}

\author{
Giuseppe Cocco ${ }^{\ddagger}$, Nader Alagha* and Christian Ibars ${ }^{\dagger, \S}$ \\ ${ }^{\ddagger}$ German Aerospace Center (DLR), Germany \\ ${ }^{\dagger}$ CTTC, Barcelona, Spain \\ ${ }^{*}$ European Space Agency, Noordwijk, The Netherlands \\ giuseppe.cocco@dlr.de, nader.alagha@esa.int, christian.ibars@cttc.es
}

\begin{abstract}
This chapter is dedicated to the application of cooperative relaying in heterogeneous land mobile satellite (LMS) systems. The aim of cooperation in this context is to help providing the missing coverage in harsh propagation environments characterized by a high node density such as urban areas. We study benefits and limits of the cooperative approach adopting a network model that is at the same time tractable and of practical interest. We derive an analytical lower bound on the coverage and show that there is a trade-off between this and the rate at which the information can be injected in the network. We also describe a possible implementation scheme for cooperative coverage extension in heterogeneous satellite LMS systems adopting the ETSI Digital Video Broadcasting - Satellite services to Handheld (DVB-SH) standard in the space segment.
\end{abstract}

\section{INTRODUCTION}

Satellite broadcasting and relaying capabilities allow to create mobile broadcast systems over wide geographical areas, which opens large market possibilities for both handheld and vehicular user terminals. Mobile broadcasting is of paramount importance for services such as digital TV or machine-to-machine (M2M) communication, a new paradigm which will bring about a tremendous increase in the number of deployed wireless terminals [1].

\footnotetext{
ฯ Giuseppe Cocco was partially founded by the CTTC and by the European Space Agency under the NPI program.

$\S$ Christian Ibars is now with Intel Corporation.
} 
Proprietary solutions as well as open standards, such as the ETSI Digital Video Broadcasting - Satellite to Handhelds (DVB-SH) [2], have been developed in the last decade to enable data broadcasting via satellite to mobile users. As of today several land mobile satellite (LMS) solutions have been already implemented for maritime and aeronautical communications [3].

Coverage, intended as the possibility for all nodes to correctly receive the data transmitted by a central node (like a satellite or a base station), is a main issue for networks with a large number of terminals. As an example, in M2M networks reliable broadcast transmission is of primary importance for terminal software and firmware update, in which all terminals need to correctly receive all the data or, for instance, navigation maps update in vehicle-mounted positioning systems. Protocols such like the Automatic Repeat-reQuest (ARQ), although very effective in point-to-point communication ([4, section 7.1.5]), may not be applicable in a multicast context due to feedback implosion issues [5]. If terminals have both mesh communication and satellite reception capabilities [6], then a cooperative approach may be viable.

A lot of work has been done on the use of cooperation in multicast and broadcast communications in both terrestrial [7][8] and satellite networks [6][9][10]. Many of the proposed solutions [5][11][12] are based on network coding [13], that can achieve the Max-flow Min-cut capacity bound in ad-hoc networks. Rateless codes have also been investigated, for instance in the context of cooperative content dissemination from road side units to vehicular networks [14] [15].

The importance of coverage extension in LMS systems stems from the fact that only terminals with an adequate channel quality are able to access satellite services and poor channel conditions frequently occur in urban areas due to the shadowing effect of surrounding obstacles, especially in case of low satellite elevation angles. In order to counteract channel impairments, terrestrial repeaters, called gap-fillers, and a link-level forward error correction LL-FEC [2] are envisaged in DVB-SH. However, the deployment of gap-fillers is very costly in terms of investment and management. A hybrid satellite-terrestrial networking approach could help to provide an adequate service level while reducing the number (or the $\cos ^{1}$ ) of the gap-fillers as we will argue later.

In the present chapter we consider the application of network coding for cooperative coverage extension in satellite broadcast channels. We carry out an analytical study on the benefits and the limits of a cooperative approach in providing missing coverage in broadcast networks. We

\footnotetext{
${ }^{1}$ the cost reduction is related to the fact that gap fillers with lower power could be used
} 
consider a mathematically tractable and yet practically interesting network model, in which fading and shadowing in the communication channels as well as the medium access mechanism of the ad-hoc network are taken into account. By applying the Max-flow Min-cut theorem we derive an analytical lower bound on the coverage as a function of both the transmission rate at physical level and the rate of innovative packets per unit-time at link level. Our results show a tradeoff between the coverage and the rate at which the information can be injected in the network, and at the same time quantify the gain deriving from cooperation, giving hints on how to tune important parameters such as the medium access probability.

We also give an example of a possible way to implement a cooperative scheme based on network coding that is compatible with existing standards, and specifically with the DVB-SH [2], which we adopt as a reference for the satellite link. We focus on vehicular terminals and adopt the IEEE $802.11 \mathrm{p}$ as reference standard for node-to-node communication. In the proposed scheme no modification is required to the DVB-SH since network coding is merged with the DVB-SH LL-FEC in the terrestrial nodes.

\section{SYSTEM MOdeL}

Let us consider a network in which a source $S$, representing the satellite (or more precisely a node generating the data broadcasted by the satellite), has a set of $K$ source messages $\boldsymbol{w}_{1}, \ldots, \boldsymbol{w}_{K}$, each of $k$ bits, to broadcast to a population of $M$ terminal nodes. Terminal nodes have both satellite reception and ad-hoc networking capabilities. No feedback is assumed from the terminals to the source and no channel state information CSI is assumed at $S$, which implies a non-zero packet loss probability. $S$ channel-encodes each message in order to decrease the probability of packet loss on the channel. Another level of protection is also applied by $S$ at packet level in order to compensate for eventual packet losses. The encoding at packet level takes place before the channel encoding. $N \geq K$ coded packets are created by $S$ applying a random linear network code (RLNC) to the $K$ source messages. We define $R=K / N$ as the rate of the network coding (NC) encoder at $S$. Network coding operates in a finite field of size $q$ $(G F(q))$, so that each message is treated as a vector of $k / \log _{2}(q)$ symbols. Source messages are linearly combined to produce encoded packets. An encoded packet $\boldsymbol{x}$ is generated as follows:

$$
\boldsymbol{x}=\sum_{i=1}^{K} \varrho_{i} \boldsymbol{w}_{i}
$$


where $\varrho_{i}, i=1, \ldots, K$ are random coefficients drawn at random according to a uniform distribution in $G F(q)$. The coefficients $\varrho_{i}, i=1, \ldots, K$, are appended to each message $\boldsymbol{x}$ before its transmission. The set of appended coefficients represents the coordinates of the encoded message $\boldsymbol{x}$ in $G F(q)$ with respect to the basis $\left\{\boldsymbol{w}_{i}\right\}, i=1, \ldots, K$, and is called global encoding vector.

The encoding at the physical layer is applied on network-encoded packets, each consisting of of $k$ bits. The transmitter encodes each packet using a Gaussian codebook of size $2^{n r}$, with $r=\frac{k}{n}$ bits per second per $\mathrm{Hz}$ (bit/s/Hz), associating a codeword $\boldsymbol{c}_{m}$ of $n$ independently and identically distributed (i.i.d.) symbols drawn according to a Gaussian distribution to each $\boldsymbol{x}_{m}$, $m=1 \ldots, N$ [4]. The time needed for $S$ to transmit a packet is called transmission slot (TS).

The terminal nodes cooperate with each other in order to recover the packets that are lost in the link from the satellite (forward link). We assume that terminals have high mobility, which is the case, for instance, in vehicular networks. In such context nodes have little time to set up a communication link with each other. For this, and in order to exploit the broadcast nature of the wireless medium, nodes act in promiscuous mode, broadcasting packets to all terminals within reach. Similarly as in the broadcast mode of IEEE 802.11 standards, no request to send (RTS)/clear to send (CTS) mechanism is assumed [16]. No CSI is assumed at the transmitter in the terminal-to-terminal communication, so that there is always a non zero probability of packet loss. Like the source, each terminal uses two levels of encoding, that are described in the following.

Let $L$ be the number of packets correctly decoded at the physical level by a terminal. The terminal selects the $L^{\prime} \leq L$ packets which constitute the largest set of linearly independent packets with respect to the basis $\boldsymbol{w}_{i}, i=1, \ldots, K$. Without loss of generality we assume that such set be $\boldsymbol{x}_{1}, \ldots, \boldsymbol{x}_{L^{\prime}}$. Linear independence is verified through the global encoding vectors of the packets. The $L^{\prime}$ packets are re-encoded together using RLNC, and then re-encoded at the physical layer. RLNC encoding at the terminals works as follows. Given the set of received packets $\boldsymbol{x}_{1}, \ldots, \boldsymbol{x}_{L^{\prime}}$, the message $\boldsymbol{y}=\sum_{m=1}^{L^{\prime}} \sigma_{m} \boldsymbol{x}_{m}$ is generated, $\sigma_{m}, m=1, \ldots, L^{\prime}$, being coefficients drawn at random according to a uniform distribution in $G F(q)$. Each time a new encoded message is created, it has its global encoding vector appended. The overhead this introduces is negligible if messages are sufficiently long [17]. The new global encoding vector 
$\boldsymbol{\eta}$ can be easily calculated by the transmitting node as follows:

$$
\eta=\sigma \Psi
$$

where $\boldsymbol{\sigma}=\left[\begin{array}{llll}\sigma_{1} & \cdots & \sigma_{L^{\prime}}\end{array}\right]$ is the local encoding vector, i.e., the vector of random coefficients chosen by the transmitting node, while $\Psi$ is an $L^{\prime} \times K$ matrix that has the global encoding vector of $\boldsymbol{x}_{m}, m=1, \ldots, L^{\prime}$, as row $m$. We assume that the transmission of a message by a terminal is completed within one TS. The physical layer encoding at a mobile node takes place in the same way as at the source, and using the same average transmission rate $r$.

\section{A. Source-to-Node Channel Model}

The channel from the source $S$ to a generic terminal $N_{i}$ (S-N channel) is affected by both Rayleigh fading and log-normal shadowing. The power of the signal received at the terminal is modeled as the product of a unit-mean exponential random variable $\gamma$ and a log-normal random variable $\Gamma_{S}$ which accounts for large scale fading. This model has been largely used to model propagation in urban scenarios [18] and, with some modifications, in LMS systems [19]. The fading coefficient $\gamma$ takes into account the fast channel variations due to the terminal motion and is assumed to remain constant within a TS, while changing in an i.i.d. fashion at the end of each channel block. The shadowing coefficient $\Gamma_{S}$ includes the transmitted power at $S$ and accounts for the obstruction of buildings in the line of sight and changes much slowly with respect to $\gamma$. For mathematical tractability we assume that $\Gamma_{S}$ remains constant for $N$ channel blocks, i.e., until all encoded packets relative to the $K$ source messages have been transmitted by $S$. We call the time needed to transmit $N$ messages a generation period $(G P)$. The fading and shadowing processes of two different nodes are assumed to be independent. We further assume that shadowing and fading statistics are the same for all nodes, which is the case if nodes are located at approximately the same distance from $S$.

A message is lost in the S-N channel if the instantaneous channel capacity is lower than the transmission rate at the physical layer $r$. Thus the packet loss probability in the S-N channel for a generic node is:

$$
P_{S N}=\operatorname{Pr}\left\{\log _{2}\left(1+\gamma \Gamma_{S}\right)<r\right\},
$$

where $\gamma \sim \exp (1)$ while $\Gamma_{S}=e^{\frac{X}{10}}$ with $X \sim \mathcal{N}\left(\mu, \sigma^{2}\right) . \Gamma_{S}$ is constant within a GP, while $\gamma$ changes independently at the end of each channel block. Fixing the value of $\Gamma_{S}$, the packet loss 
probability $P_{S N}$ in the $\mathrm{S}-\mathrm{N}$ link is:

$$
P_{S N}=1-e^{\frac{1-2^{r}}{\Gamma_{S}}}
$$

In the rest of the chapter we will use the expressions "packet loss rate" and "probability of packet loss" interchangeably. Due to shadowing, $\Gamma_{S}$ changes randomly and independently at each generation period and, within a generation, from one node to the other. Thus the packet loss rate $P_{S N}$ is also a random variable that remains constant within a generation and changes in an i.i.d. fashion across generations and terminals.

\section{B. Node-to-Node Channel Model}

We model the channels between the transmitting terminal and each of the receiving terminals (N-N channel) as independent block fading channels, i.e., the fading coefficient of each channel changes in an i.i.d. fashion at the end of each channel block. The probability of packet loss in the N-N channel $P_{N N}$ is:

$$
P_{N N}=\operatorname{Pr}\left\{\log _{2}\left(1+\gamma \Gamma_{N}\right)<r\right\}=1-e^{\frac{1-2^{r}}{\Gamma_{N}}},
$$

where $\Gamma_{N}$ accounts for path loss and transmitted power, and is assumed to remain constant for a whole generation period and across terminals. In order not to saturate the terrestrial channel, we assume that a node can transmit at most one packet within one TS. Note that $P_{N N}$ (unlike $\left.P_{S N}\right)$ is not a random variable since $\Gamma_{N}$ is a deterministic constant.

\section{NON-COOPERATIVE SCENARIO}

Let us consider a network with a source $S$ and $M$ terminals. We define the coverage $\Omega$ as the probability that all $M$ terminals correctly decode the whole set of $K$ source messages ${ }^{2}$. Assuming $K$ large enough and using the results in [5], the probability that node $N_{i}$ can decode all the $K$ source messages of a given generation in case of no cooperation is:

$$
\operatorname{Pr}\left\{P_{S N i}<1-R\right\}=F_{P_{S N i}}(1-R),
$$

$F_{P_{S N}}$ being the cumulative density function (cdf) of $P_{S N}$ and $R=K / N$ being the rate of the NC encoder at $S$. We recall that, due to the shadowing, the packet loss rate $P_{S N}$ is a random

\footnotetext{
${ }^{2}$ for correctness we point out that this is a slight misuse of the term "coverage", since in satellite communications the term has usually a geographical connotation.
} 
variable which changes in an i.i.d. fashion across generations and terminals. Plugging Eqn. (2) into Eqn. (4) we find:

$$
\operatorname{Pr}\left\{1-e^{\frac{1-2^{r}}{\Gamma_{S}}}<1-R\right\}
$$

The coverage, intended as the probability that each of the nodes decodes all source messages, is:

$$
\Omega=\operatorname{Pr}\left\{P_{S N 1}<1-R, \ldots, P_{S N M}<1-R\right\}
$$

where $P_{S N i}$ is the packet loss rate in the S-N link of node $N_{i}, i=1, \ldots, M$. Under the assumption of i.i.d. channels we have $F_{P_{S N i}}=F_{P_{S N}}, \forall i \in\{1, \ldots, M\}$. Thus Eqn. (6) can be written as:

$$
\Omega=\left(\operatorname{Pr}\left\{P_{S N}<1-R\right\}\right)^{M}=F_{P_{S N}}^{M}(1-R),
$$

$F_{P_{S N}}(y)$ being the cdf of $P_{S N}$, which can be obtained as follows.

Let us rewrite the log-normal variable $\Gamma_{S}$ as: $\Gamma_{S}=e^{\frac{X}{10}}$, where $X \sim \mathcal{N}\left(\mu, \sigma^{2}\right)$. Fixing the variable $X$ the packet loss rate $P_{S N}=Y$ is:

$$
Y=1-e^{\left(1-2^{r}\right) \cdot e^{-\frac{X}{10}}} .
$$

The cdf of $Y$ can be derived as:

$$
\begin{aligned}
F_{Y}(y) & =\operatorname{Pr}\{Y<y\} \\
& =\operatorname{Pr}\left\{1-e^{\left(1-2^{r}\right) \cdot e^{-\frac{X}{10}}}<y\right\} \\
& =\operatorname{Pr}\left\{\ln (1-y)<\left(1-2^{r}\right) \cdot e^{-\frac{X}{10}}\right\} \\
& =\operatorname{Pr}\left\{X>10 \ln \left[\frac{1-2^{r}}{\ln (1-y)}\right]\right\} \\
& =1-F_{X}\left(10 \ln \left[\frac{1-2^{r}}{\ln (1-y)}\right]\right) \\
& =\frac{1}{2}-\frac{1}{2} \operatorname{erf}\left(\frac{10 \ln \left[\frac{1-2^{r}}{\ln (1-y)}\right]-\mu}{2 \sigma^{2}}\right),
\end{aligned}
$$

for $y \in(0,1)$, where $\operatorname{erf}(x)$ is the error function, defined as $\frac{2}{\sqrt{\pi}} \int_{0}^{x} e^{-t^{2}} d t$.

Finally, plugging Eqn. (8) into Eqn. (7), we find the coverage in the non cooperative case:

$$
\Omega=\frac{1}{2^{M}}\left[1-\operatorname{erf}\left(\frac{10 \ln \left[\frac{1-2^{r}}{\ln (R)}\right]-\mu}{2 \sigma^{2}}\right)\right]^{M}
$$


for $R \in(0,1)$. Note that, fixing $R$ and $M$, the expression in Eqn. (8) goes to 0 as the rate at physical level $r$ goes to infinity (or, mutatis mutandis, fixing $r$ and letting $R$ go to 1 the coverage goes to zero). This confirms the intuition that the coverage decreases as the transmission rate increases. As said previously this result holds for any value of $q$ as long as $K$ is large enough. Thus, Eqn. (8) can also be interpreted as the coverage in a network of $M$ nodes in presence of fading and shadowing that can be achieved using a rateless code over $G F(2)$ with rate $R$.

\section{CoOperative Scenario}

The wireless network is modeled as a directed hypergraph $\mathcal{H}=(\mathcal{N}, \mathcal{A}), \mathcal{N}$ being a set of nodes and $\mathcal{A}$ a set of hyperarcs. A hyperarc is a pair $(i, J)$, where $i$ is the head node of the hyperarc while $J$ is the tail, i.e., the subset of $\mathcal{N}$ connected to the head through the hyperarc. A hyperarc $(i, J)$ can be used to model a broadcast transmission from node $i$ to nodes in $J$. Packet losses can be taken into account. Our goal is to derive the relationship between the coverage and the rate at which the information is transferred to the mobile terminals, which depends on both the rate at physical level $r$ and the rate at which new messages are injected in the network, i.e., the rate at packet level $R$. In [5] (Theorem 2) it is shown that, if $K$ is large, random linear network coding achieves the network capacity in wireless multicast and unicast connections, even in case of lossy links, if the number of innovative packets transmitted by the source per unit of time is lower than or equal to the flow across the minimum flow cut between the source and each of the sink nodes. This can be expressed mathematically as:

$$
R \leq \min _{Q \in \mathcal{Q}(S, t)}\left\{\sum_{(i, J) \in \Gamma_{+}(Q)} \sum_{T \nsubseteq Q} z_{i J T}\right\}
$$

where $z_{i J T}$ is the average injection rate of packets in the arcs departing from $i$ to the tail subset $T \subset J, \mathcal{Q}(S, t)$ is the set of all cuts between $S$ and $t$, and $\Gamma_{+}(Q)$ denotes the set of forward hyperarcs of the cut $Q$, i.e.:

$$
\Gamma_{+}(Q)=\{(i, J) \in \mathcal{A} \mid i \in Q, J \backslash Q \neq 0\} .
$$

In other words, $\Gamma_{+}(Q)$ denotes the set of arcs of $Q$ for which the head node is on the same side as the source, while at least one of the tail nodes of the relative hyperarc belongs to the other side of the cut. The rate $z_{i J T}$ is defined as:

$$
z_{i J T}=\lim _{\tau \rightarrow \infty} \frac{A_{i J T}(\tau)}{\tau},
$$


where $A_{i J T}(\tau)$ is a process representing the number of packets sent by $i$ that arrive in $T \subset J$ in the temporal interval $[0, \tau)$. The existence of an average rate is a necessary condition for the applicability of the results in [5].

In the following we derive $z_{i J T}$ for the considered network setup as a function of both physical layer and MAC layer parameters such as transmission rate, transmission power and medium access probability.

\section{A. Medium Access}

Let us consider a network with $M$ nodes. We assume that all nodes have independent $\mathrm{S}-\mathrm{N}$ and $\mathrm{N}-\mathrm{N}$ channels. We further assume that channel statistics are the same for all terminals (i.e., all $\mathrm{N}-\mathrm{N}$ channels have the same statistics and all the S-N channels have he same statistics, possibly different by the N-N channels), which is the case if the distances from node $N_{i}$ to node $N_{j}$ change little $\forall i, j \in\{1, \ldots, M\}, i \neq j$ and with respect to each node's distance to the source.

In our setup the terminals are set in promiscuous mode so that each node can overhear the broadcast transmissions of any other node [16]. The terminals share the wireless medium, i.e., they transmit in the same frequency band. We assume that a CSMA/CA protocol is adopted by the nodes and that all nodes hear each other, so that the medium is shared among the terminals willing to transmit but no collision happens.

We now derive an expression for the communication rate $z_{i J T}$. We start by deriving the communication rate $z_{i j}$ between a transmitting node $N_{i}$ and a single receiving node $N_{j}$. By the symmetry of the problem all links have the same average rate. Consider the generic transmitting node $N_{i}$. The average transmission rate from node $N_{i}$ to node $N_{j}$ is:

$$
\begin{aligned}
z_{i, j} & =p_{a} \cdot \operatorname{Pr}\{\text { No one else transmits }\}\left(1-P_{N N}\right) \\
& =p_{a} \cdot\left[\operatorname{Pr}\{\text { No one else tries to transmit }\}+\operatorname{Pr}\left\{N_{i} \text { wins contention }\right\}\right]\left(1-P_{N N}\right)(12)
\end{aligned}
$$

where $p_{a}$ is the probability that a node tries to contend for the channel. We assume, for mathematical tractability, that $p_{a}$ is fixed for all nodes. The first term in the sum of Eqn. (12) is:

$$
\operatorname{Pr}\{\text { No one else tries to transmit }\}=\left(1-p_{a}\right)^{M-1} .
$$

The second term in the sum of Eqn. (12) is the probability that one or more other nodes contend for the channel, but $N_{i}$ transmits first. To calculate this probability, we note that, if $k$ other nodes 
try to access the channel (for a total of $k+1$ nodes contending for the channel), the probability for each of them to occupy the channel before the others is $1 /(k+1)$. Thus we can write:

$$
\begin{aligned}
\operatorname{Pr}\left\{N_{i} \text { wins contention }\right\} & =\sum_{k=1}^{M-1}\left(\begin{array}{c}
M-1 \\
k
\end{array}\right) \frac{p_{a}^{k}\left(1-p_{a}\right)^{M-1-k}}{k+1} \\
& =\frac{1}{M p_{a}} \sum_{k=1}^{M-1}\left(\begin{array}{c}
M \\
k+1
\end{array}\right) p_{a}^{k+1}\left(1-p_{a}\right)^{M-1-k} \\
& =\frac{1}{M p_{a}} \sum_{k=2}^{M}\left(\begin{array}{c}
M \\
k
\end{array}\right) p_{a}^{k}\left(1-p_{a}\right)^{M-k} \\
& =\frac{1}{M p_{a}}\left[1-\left(\begin{array}{c}
M \\
0
\end{array}\right)\left(1-p_{a}\right)^{M}-\left(\begin{array}{c}
M \\
1
\end{array}\right) p_{a}\left(1-p_{a}\right)^{M-1}\right] \\
& =\frac{1}{M p_{a}}\left[1-\left(1-p_{a}\right)^{M}-M p_{a}\left(1-p_{a}\right)^{M-1}\right] .
\end{aligned}
$$

Plugging equations (13) and (14) into Eqn. (12) we obtain:

$$
z_{i, j}=\frac{1-\left(1-p_{a}\right)^{M}}{M}\left(1-P_{N N}\right) .
$$

Using the definition given by Eqn. (11) together with Eqn. (15), we finally find

$$
z_{i J T}=\frac{1-\left(1-p_{a}\right)^{M}}{M}\left[1-\left(P_{N N}\right)^{|T|}\right]
$$

where $|T|$ is the cardinality of $T$, and the term $\left[1-\left(P_{N N}\right)^{|T|}\right]$ is the probability that at least one of the $|T|$ nodes whose S-link belongs to the cut receives correctly a transmission from a node that is in the other side of the cut. Expression (16) can be interpreted as the rate at which packets are received by the set $T$ considered as a single node, that is, the counting process $A_{i J T}(\tau)$ increases by one unit when at least one of the terminals in $T$ receives one packet, independently from the actual number of terminals that received it.

\section{B. Coverage Analysis}

In the following we derive the condition that maximizes the coverage as a function of relevant network parameters by applying the Max-flow Min-cut theorem [20]. We recall that such maximum coverage can be attained by using the random coding scheme described in Section II.

Let us consider Eqn. (9). For each of the $M$ nodes we must consider all the possible cuts of the network such that the node and the satellite are on different sides of the cut. Let us fix a 
receiving node $N_{t}$. We recall that a cut is a set of edges that, if removed from a graph, separates the source from the destination. Fig. 1 gives an example of a network with four nodes where the cut $Q_{S N_{4}}$ (i.e., the cut such that $N_{4}$ and $S$ are on the same side) is put into evidence. In the example, the destination node is $N_{t}=N_{1}$. The dotted lines represent the edges which are to be removed in order to get the cut. Note that the set of nodes foe which the satellite link is preserved (only node $N_{4}$ in the figure) are isolated by the cut from the nodes with satellite cut (nodes $N_{1}, N_{2}$ and $N_{3}$ in Fig. 1). We define a satellite edge (S-edge) as an edge of the kind $\left(S, N_{j}\right), j \neq t$. We further define a terrestrial-edge (T-edge) as one of the kind: $\left(N_{j}, N_{t}\right), j \neq t$. First of all, we note that in each possible cut of $N_{t}=N_{1}$ the arc joining the node with the

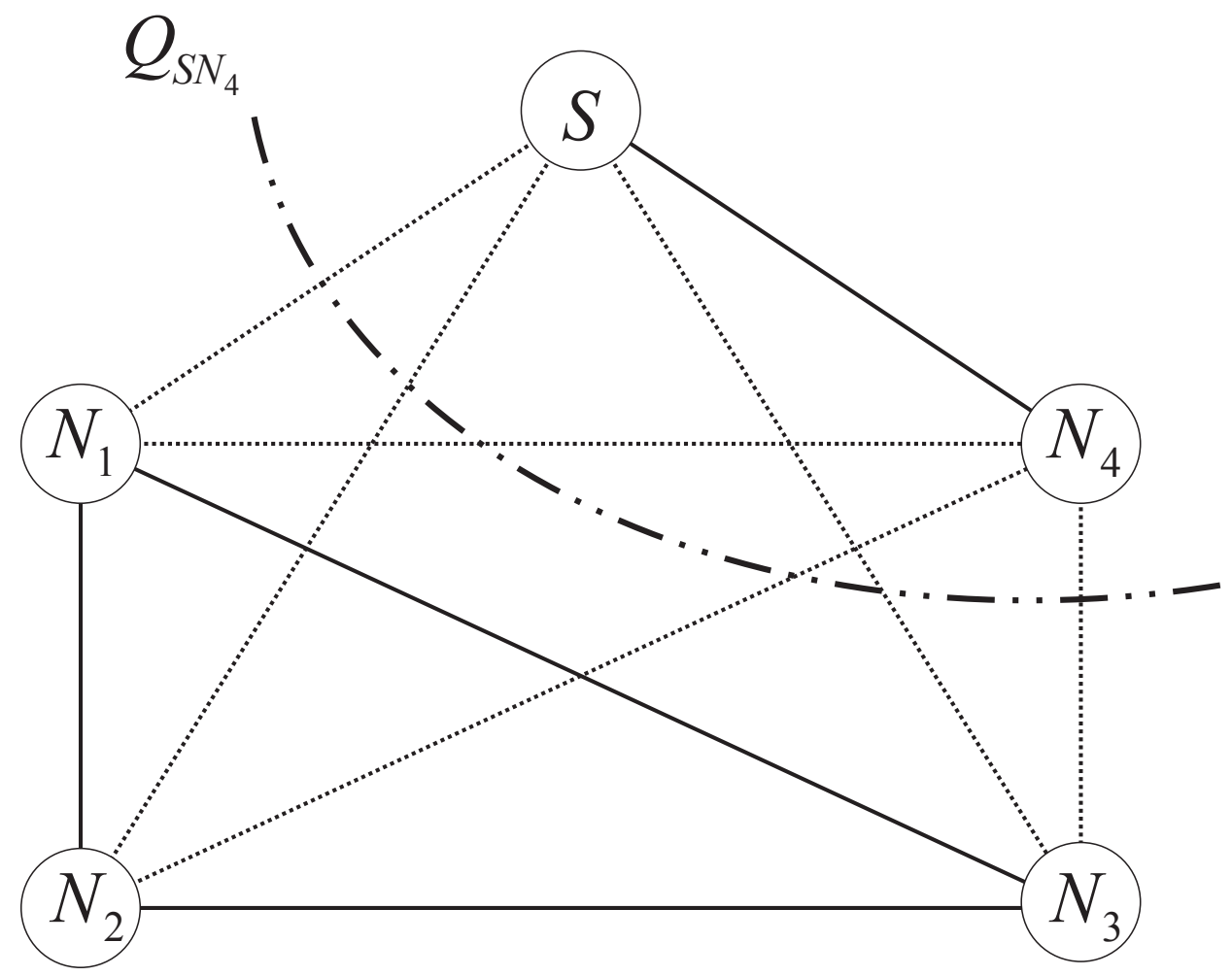

Fig. 1. Graph model of a network with four terminals. The number of possible cuts for each of the $M$ nodes is $2^{M-1}=8$. The set of nodes that receive from $S$ (only node $N_{4}$ in the figure) are isolated by the cut from the nodes with satellite cut (i.e., nodes whose S-N link is removed from the cut).

source is always present. For the particular network topology considered, the rest of the cuts are obtained by removing, for each of the $M-1$ remaining nodes, either the S-link or the T-link between the considered node and $N_{t}$. The number of possible cuts is thus equal to $2^{M-1}$. Two 
distinct cuts differ in either the number $n_{s}$ of S-edges which are included in the cut or the identity of the nodes for which the S-edge is part of the cut. For each $N_{t} \in \mathcal{N}$ and for each cut such that $n_{s} \in\{1, \cdots, M-1\}$ S-links are present, the average message rate $R$ at the source must be lower than or equal to the capacity of the cut, i.e.:

$$
R \leq 1-\prod_{j \in Q_{n_{s}}} Y_{j}+\left(M-n_{s}\right) \frac{1-\left(1-p_{a}\right)^{M}}{M}\left[1-\left(P_{N N}\right)^{n_{s}}\right],
$$

that can be rewritten as

$$
\alpha\left(n_{s}\right)-\prod_{j \in Q_{n_{s}}} Y_{j} \geq 0,
$$

where $Q_{n_{s}}$ is one of the cuts with $n_{s}$ satellite links relative to the node $N_{t}$ and we defined:

$$
\alpha\left(n_{s}\right)=1-R+\left(M-n_{s}\right) \frac{1-\left(1-p_{a}\right)^{M}}{M}\left[1-\left(P_{N N}\right)^{n_{s}}\right] .
$$

The right hand term of Eqn. (17) can be decomposed into two terms. One is

$$
1-\prod_{j \in Q_{n_{s}}} Y_{j}
$$

that can be interpreted as the amount of information that reaches the set of nodes with satellite cut considered as a single entity (or alternatively the probability that at least one of the nodes with satellite cut correctly receives a given packet). The second term is

$$
\left(M-n_{s}\right) \frac{1-\left(1-p_{a}\right)^{M}}{M}\left[1-\left(P_{N N}\right)^{n_{s}}\right]
$$

that can be interpreted as the information that flows from the $M-n_{s}$ nodes on the satellite side of the cut to the set of $n_{s}$ nodes on the other side of the cut considered as a single entity. This last term is the contribution introduced by the cooperation.

The condition in Eqn. (18) must hold for any number $n_{s}$ of S-edges. This is equivalent to imposing a new condition which is the intersection of all the conditions of the kind of Eqn. (18), i.e.:

$$
\bigcap_{Q_{n_{s}} \in \mathcal{S}\left(n_{s}, \bar{N}_{t}\right)}\left[\prod_{j \in Q_{n_{s}}} Y_{j} \leq \alpha\left(n_{s}\right)\right]
$$

where $\mathcal{S}\left(n_{s}, \bar{N}_{t}\right)$ is the set of all subsets of $\mathcal{N} \backslash N_{t}$ with $n_{s}$ elements. The number of elements in $\mathcal{S}\left(n_{s}, \bar{N}_{t}\right)$ is $\left(\begin{array}{c}M-1 \\ n_{s}\end{array}\right)$, as each of them is obtained by choosing $n_{s}$ elements from a set with cardinality $M-1$. As we mentioned previously, for a given $N_{t}$ to decode all messages the 
condition on the flow must be satisfied across all cuts, which is equivalent to imposing the condition given by expression (19) for all $n_{s}$. Finally, in order for all nodes to decode all source messages the condition on the minimum flow cut must hold $\forall t \in \mathcal{N}$. Imposing this, we obtain the expression for the coverage that is reported in Eqn. (20) at the bottom of the page.

\section{Lower Bound on Achievable Coverage}

Although Eqn. (20) might be used to evaluate $\Omega$ numerically, a closed-form expression would give more insight into the impact of cooperation on the considered setup. Finding a simple closed form expression for Eqn. (20) is a challenging task. Thus in the following we derive a lower bound $\Omega_{L B}$ on $\Omega . \Omega$ can be lower bounded by substituting in Eqn. (20) the packet loss rate $Y_{j}$ for each cut with the largest packet loss rate among all the S-links in the network, i.e.:

$$
\begin{aligned}
\Omega & =\operatorname{Pr}\left\{\bigcap_{N_{t} \in \mathcal{N}} \bigcap_{n_{s} \in\{1, \ldots, M\}} \bigcap_{Q_{n_{s}} \in \mathcal{S}\left(n_{s}, \bar{N}_{t}\right)}\left[\prod_{j \in Q_{n_{s}}} Y_{j}<\alpha\left(n_{s}\right)\right]\right\} \\
& \geq \operatorname{Pr}\left\{\bigcap_{N_{t} \in \mathcal{N}} \bigcap_{n_{s} \in\{1, \ldots, M\}}\left[\prod_{j=1}^{n_{s}} Y_{(j)}<\alpha\left(n_{s}\right)\right]\right\} \\
& \geq \operatorname{Pr}\left\{\bigcap_{\bigcap_{t} \in \mathcal{N}} \bigcap_{n_{s} \in\{1, \ldots, M\}}\left[Y_{(1)}^{n_{s}}<\alpha\left(n_{s}\right)\right]\right\} \\
& =\operatorname{Pr}\left\{\bigcap_{\bigcap_{t} \in \mathcal{N} n_{s} \in\{1, \ldots, M\}}\left[Y_{(1)}<\sqrt[n_{s}]{\alpha\left(n_{s}\right)}\right]\right\} \\
& =\operatorname{Pr}\left\{Y_{(1)}<\min _{n_{s} \in\{1, \ldots, M\}} \sqrt[n_{s}]{\alpha\left(n_{s}\right)}\right\} \\
& =F_{Y}^{M}(\beta),
\end{aligned}
$$

$\Omega=\operatorname{Pr}\left\{\bigcap_{N_{t} \in \mathcal{N}} \bigcap_{n_{s} \in\{1, \ldots, M-1\}} \bigcap_{Q_{n_{s}} \in \mathcal{S}\left(n_{s}, \bar{N}_{t}\right)}\left[\prod_{j \in Q_{n_{s}}} Y_{j}<1-R+\left(M-n_{s}\right) \frac{1-\left(1-p_{a}\right)^{M}}{M}\left[1-\left(P_{N N}\right)^{n_{s}}\right]\right]\right\}$. 
where $Y_{(i)}$ is the $i$-th largest packet loss rate across all S-edges of the network, i.e., $Y_{(i)} \geq Y_{(j)}$ if $i<j, \forall i, j \in \mathcal{N}$, and we defined

$$
\beta=\min _{n_{s} \in\{1, \ldots, M\}} \sqrt[n_{s}]{\alpha\left(n_{s}\right)}
$$

Inequality (21) derives from the fact that:

$$
\prod_{j \in S} Y_{j} \leq \prod_{j=1}^{n_{s}} Y_{(j)}, \text { for } S \in \mathcal{S}\left(n_{s}, \bar{t}\right), \forall n_{s}, t,
$$

i.e., we substitute the product of $n_{s}$ random variables, chosen within a set of $M$ variables, with the product of the $n_{s}$ largest variables of the same set. Inequality (22) follows from the fact that

$$
\prod_{j=1}^{n_{s}} Y_{(j)} \leq Y_{(1)}^{n_{s}}, \forall n_{s}, t .
$$

By plugging Eqn. (8) into Eqn. (21) we finally find:

$$
\Omega_{L B}=\frac{1}{2^{M}}\left[1-\operatorname{erf}\left(\frac{10 \ln \left[\frac{1-2^{r}}{\ln (1-\beta)}\right]-\mu}{2 \sigma^{2}}\right)\right]^{M} .
$$

Example: A Two-nodes Network: In order to clarify the concepts just described, in the following we consider the case of a network with only two nodes, such as the one depicted in Fig. 2. We start by deriving the communication rates over the terrestrial edge. In each slot node $N_{i}$ tries to access the channel with probability $p_{a i}$. In case only node $N_{i}$ tries to access the channel, the transmission will be successful with probability $1-P_{N N}$, where $P_{N N}$ is the packet loss probability in the link between the two nodes. In case both nodes try to access the channel in the same slot, the CSMA/CA mechanism determines which of the two nodes transmits. Given the symmetry of the problem, in case of contention each of the two nodes occupies the channel with probability $1 / 2$ and the transmission is successfully received by the other node with probability $1-P_{N N}$. According to Eqn. (14), the average rate on the edge $\left(N_{1}, N_{2}\right)$ can be written as:

$$
z_{1,2}=p_{a 1}\left[\left(1-p_{a 2}\right)\left(1-P_{N N}\right)+\frac{p_{a 2}}{2}\left(1-P_{N N}\right)\right]=p_{a 1}\left(1-\frac{p_{a 2}}{2}\right)\left(1-P_{N N}\right),
$$

while

$$
z_{2,1}=p_{a 2}\left(1-\frac{p_{a 1}}{2}\right)\left(1-P_{N N}\right)
$$

With reference to Fig. 2, the cuts in the network graph are: $Q_{S}$ in which the satellite and the nodes lie in different sides of the cut, $Q_{S N_{1}}$, in which node $N_{1}$ is on the satellite side and $Q_{S N_{2}}$, 


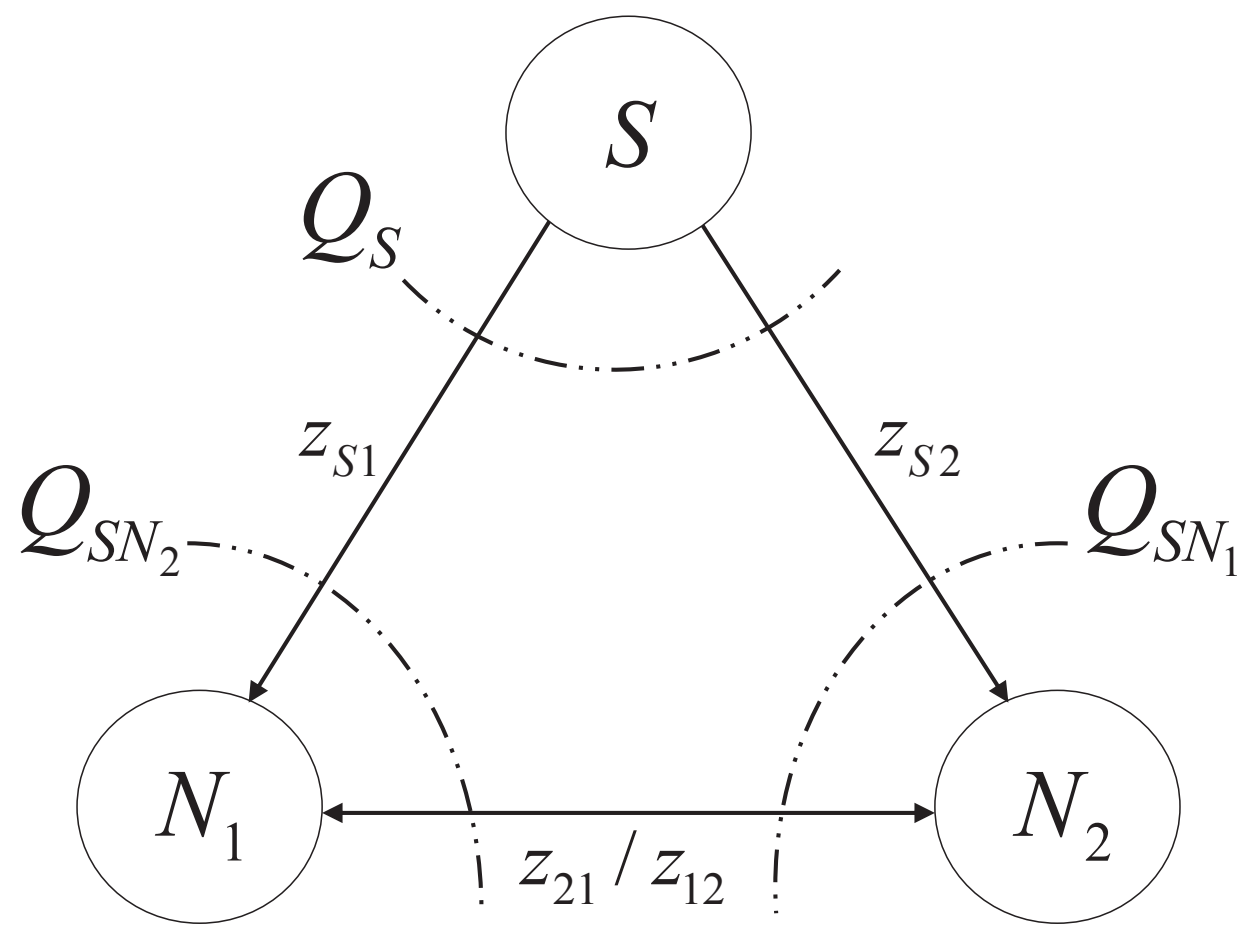

Fig. 2. Graph model for a network with two nodes. $Q_{S}, Q_{S N_{1}}$ and $Q_{S N_{2}}$ are the three cuts of the network. $Q_{S}$ is the cut in which the satellite and the nodes lie in different sides, $Q_{S N_{1}}$ is the cut in which node $N_{1}$ is on the satellite side and $Q_{S N_{2}}$ is the cut in which node $N_{2}$ is on the satellite side. $z_{i j}$ is the average injection rate in the edge $(i, j)$.

in which node $N_{2}$ is on the satellite side. The conditions on the flows across the three cuts are:

$$
\begin{aligned}
& Q_{S}: 1-P_{L S 1} \cdot P_{L S 2} \geq R \\
& Q_{S N_{1}}: 1-P_{N N 2}+p_{a 2}\left(1-p_{a 1}\right)\left(1-P_{N N}\right) \geq R \\
& Q_{S N_{2}}: 1-P_{N N 1}+p_{a 1}\left(1-p_{a 2}\right)\left(1-P_{N N}\right) \geq R .
\end{aligned}
$$

Hence the maximum achievable rate $R^{*}$ is:

$$
R^{*}=\min \left\{1-P_{N N 1} \cdot P_{N N 2}, 1-P_{N N 2}+p_{a 2}\left(1-p_{a 1}\right)\left(1-P_{N N}\right), 1-P_{N N 1}+p_{a 1}\left(1-p_{a 2}\right)\left(1-P_{N N}\right)\right\} .
$$

Note that in Eqn. (27) $P_{L S 1}$ and $P_{L S 2}$ are i.i.d. random variables, and thus also $R^{*}$ is a random variable. As the pair $(r, R)$ is fixed, there is a nonzero probability that $R>R^{*}$, i.e., the packet injection rate at the satellite is not supported, which implies that either one or both the terminals are not able to recover all source packets. By definition of coverage we have:

$$
\Omega=\operatorname{Pr}\left\{R^{*} \geq R\right\}
$$


If we impose $p_{a 1}=p_{a 2}=p_{a}$ we have $z_{1,2}=z_{2,1}$. According to the notation defined in previous subsection we define

$$
\begin{array}{r}
Y_{(1)}=\max \left\{P_{N N 1}, P_{N N 2}\right\}, \\
Y_{(2)}=\min \left\{P_{N N 1}, P_{N N 2}\right\}, \\
\alpha(1)=1-R+p_{a 1}\left(1-\frac{p_{a 2}}{2}\right)\left(1-P_{N N}\right) \\
=1-R+\frac{1-\left(1-p_{a}\right)^{2}}{2}\left(1-P_{N N}\right),
\end{array}
$$

and

$$
\alpha(2)=1-R
$$

Finally, applying Eqn. (21) we derive the following lower bound on $\Omega$ for a network with 2 nodes:

$$
\Omega \geq F_{Y}^{2}(\min \{\alpha(1), \sqrt{\alpha(2)}\})
$$

\section{Cooperative Coverage Extension in DVB-SH}

In the following we describe a possible way to apply the cooperative approach described in the previous section in heterogeneous satellite vehicular networks.

\section{A. Space Segment}

1) Satellite Channel: The considered setup is an LMS system with a GEO satellite in L band (or low $\mathrm{S}$ band) broadcasting a DVB-SH-B signal to a population of mobile terminals. In DVBSH-B an OFDM waveform is used at the gap-fillers while a non-OFDM (usually called TDM) signal is used at the satellite. Propagation conditions depend on the presence of buildings and trees and are classified in urban, suburban and rural. The main cause of channel impairment in urban and suburban environments is the long-lasting shadowing caused by the buildings, which translates in intermittent satellite connectivity, while in the rural propagation scenarios the main source of impairment is tree shadowing. 
2) MPE-IFEC in DVB-SH: In order to counteract the harsh propagation conditions of Urban and Suburban environments, two levels of protection are envisaged in DVB-SH. One is applied at the physical layer, which includes a long physical-layer interleaver and powerful channel codes, while the other is applied at a higher layer. Such high-level protection is referred to as the Multi-Protocol Encapsulation-Inter-burst Forward Error Correction (MPE-IFEC), and is meant to provide an alternative to the long physical layer interleaver. The MPE-IFEC is a process section between the IP and the transport layers introduced in DVB-SH in order to counteract the disturbances in reception and transmission. This is achieved by applying FEC over multiple groups of datagrams called datagram bursts. The long high-layer interleaver used in IFEC allows for significant performance enhancements with respect to FEC [2], as it can better counteract long-lasting shadowing.

The encoding is made over several datagram bursts. Each datagram burst entering the MPEIFEC process is reshaped in a matrix of $T$ by $C$ bytes called Application Data Sub-Table (ADST) illustrated in Fig. 3 [2]. The columns of the ADST are then distributed in a round robin fashion

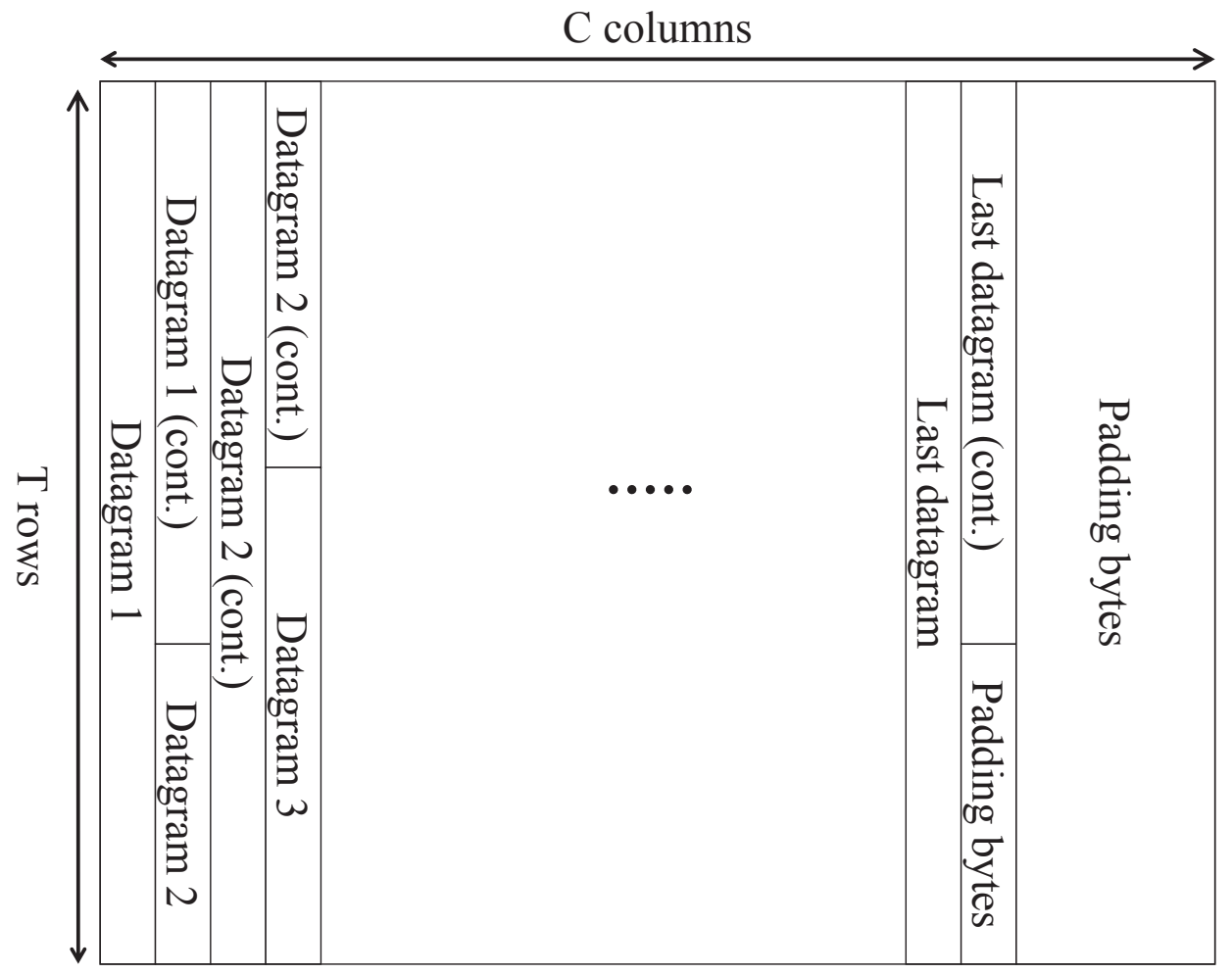

Fig. 3. ADST reshaping of datagram bursts. 
among $B$ matrices called Application Data Tables (ADT). An ADT is a $T$ by $K$ matrix. The FEC, always systematic, is applied on the ADT producing a $T$ by $N_{r}$ parity matrix, called IFEC Data Table (iFDT). An ADT is filled up and the encoding takes place every EP bursts, EP being the Encoding Period, which determines the number of datagram bursts over which the parity is calculated. The ADT and the iFDT together form an encoding matrix. It takes $B \times E P$ bursts to fill up a single ADT. Once an ADT is full (this happens to $B$ ADT at the same time) the iFDT is calculated. As soon as the $B$ iFDTs are calculated an IFEC burst is generated by taking groups of columns from $S$ different iFDTs. An IFEC burst is made up of several IFEC sections. Each section is comprised of a header, a payload containing $g$ columns from the same iFDT and a cyclic redundancy check (CRC). The $k$-th IFEC burst is merged with the $(k-D)$-th datagram burst (and eventual MPE-FEC redundancy) to form a time-slice burst. The time slice burst is then multiplexed on MPEG2-TS frames and passed down to lower layers.

Depending on the FEC technique applied (Reed-Solomon or Raptor), different values of $E P$, $B$ and $S$ are adopted. In case a Raptor code is used EP is generally greater than 1 , while $B=S=1$. This is because Raptor codes, unlike other FEC codes such as Reed-Solomon codes [21], are capable of handling large source matrices (i.e., ADT), that can span several datagram bursts.

a) Raptor Codes in DVB-SH: The Raptor code adopted for the DVB-SH is the same as in the 3GPP standard, which has also been adopted in the DVB-Handheld (DVB-H) standard [2]. Its description can be found in [22]. A source block in [22] corresponds to an ADT and a source symbol is a column of the ADT. Thus a source block has $K$ symbols of $T$ bytes each. The Raptor encoder is applied independently to each source block, each of which is identified by a Source Block Number (SBN). The encoder produces $K$ systematic symbols (the ADT matrix) and $N_{r}$ repair (parity) symbols. Systematic and repair symbols are called encoding symbols. Each symbol is identified by an Encoding Symbol Identifier (ESI). Values from 0 to $K-1$ are assigned to the systematic symbols, while values from $K$ to $N_{r}+K-1$ identify repair symbols. The encoding procedure consists of two parts. In the first part $L$ intermediate symbols are produced starting from the $K$ source symbols, while in the second part $K+N_{r}$ encoding symbols are generated starting from the $L$ intermediate symbols.

The intermediate symbols from 0 to $K-1$ are systematic (i.e., they are the same as the source symbols). The $S$ intermediate symbols from $K$ to $K+S-1$ are generated using an 
LDPC encoder while the last $H$ symbols from $K+S$ to $L$ are called Half Symbols and are generated using a binary reflected Gray encoder [2].

The encoding symbols are generated applying a Luby Transform (LT) encoder to the $L$ intermediate symbols. The LT encoder operates a bit-wise XOR of intermediate symbols chosen according to a certain degree distribution. Each of the encoding symbols is transmitted together with its ESI and a triple $(d, a, b)$ where $d$ is the symbol degree and $a$ and $b$ are integers from the sets $1, \ldots, L^{\prime \prime}-1$ and $0, \ldots, L^{\prime \prime}-1$ respectively, $L^{\prime \prime}-1$ being the smallest prime integer greater than or equal to $L$. At the end of the encoding process, $K$ systematic symbols plus $N_{r}$ parity symbols are produced. The parity symbols are linear combinations of systematic symbols in $G F(2)$. The encoding symbol triple together with the ESI and the value $K$ allows the decoder to determine which intermediate symbols (and thus which source symbols) were combined to form each of the encoding symbols.

\section{B. Ground Segment}

We consider high class terminals as defined in [23]. High class terminals are (almost) not energy constrained and have relatively good computation capabilities and memory [23]. This is the case with vehicular terminals that are powered by rechargeable batteries and can host highly performant computation units thanks to the relative low impact they have in terms of cost, space and weight. We assume that each terminal has both satellite and ad-hoc networking capabilities.

More specifically we assume that each vehicle is equipped with a DVB-SH receiving terminal for satellite signal reception. As for the node-to-node communication we consider the use of the Dedicated Short Range Communication (DSRC)/IEEE 802.11p standard which is specific for vehicle-to-vehicle communication $(\mathrm{V} 2 \mathrm{~V})$ in the $5.9 \mathrm{GHz}$ band. However, note that the proposed cooperation method is transparent to the standard used for the $\mathrm{V} 2 \mathrm{~V}$ channel, and thus different solutions could be adopted.

\section{NETWORK-CODED COOPERATION FOR DVB-SH}

In the following we give an example of a cooperative scheme for coverage enhancement in the forward link [24]. We call such cooperation scheme Network-coded Cooperative Coverage Enhancement (NCCE). Let us consider a satellite broadcasting a DVB-SH-B signal with MPEIFEC protection to a population of vehicular terminals with both DVB-SH-B and IEEE 802.11p 
radio interfaces. During a time window $(0, t)$ the satellite transmits $K+N_{r}$ IFEC symbols obtained from an ADT. Terrestrial and satellite communications take place in orthogonal frequency bands. Due to long-lasting shadowing caused by urban propagation conditions, it can happen that a user decodes a number of symbols equal to $M<K$ during the interval $(0, t)$. In this case the user cannot decode the entire source data block. In order to enhance satellite coverage each node re-encodes the received packets (either received directly from the satellite or from other terminals) and broadcasts them to nodes within its transmission range. In the following sections we describe the encoding procedure at land mobile nodes.

\section{A. Encoding at Land Mobile Nodes}

Let us assume that a node is able to decode some of the encoding symbols directly from the satellite. Each symbol carries an ESI and a triple $(d, a, b)$. As described in Section V-A2 the node uses the ESI to understand which of the source symbols were combined together to form the considered encoding symbol. We propose to apply a network encoding scheme at land mobile nodes using the source symbols of iFEC as source symbols of the network code. In other words, nodes exchange linear combinations of encoding symbols in some finite field, with the aim of recovering all the source symbols.

\section{B. Terrestrial Channel Usage}

Each received encoding symbol is interpreted by a node as a linear combination of source symbols with coefficients 0 or 1 in $G F\left(2^{n}\right)$, where $n$ is an integer corresponding to the number of bits used to represent each coefficient. The node then applies the network encoding procedure described in Section II. The encoding vector of the received encoding symbol can be derived from symbol's ESI and triple $(d, a, b)$.

The probability to access the channel in each slot is determined by the parameter cooperation level which we indicate with $\zeta, 0 \leq \zeta \leq 2$. In the following we will assume that $\zeta$ is the same for all nodes. Fixing $\zeta \leq 1$, in each slot, if a node stored a number of linearly independent packets which is larger than the number of transmitted packets in the current generation, it creates a linear combination of all the stored packets as described in Section IV and tries to access the channel with probability $\zeta$. If $\zeta>1$ two cases must be considered. In case the number of transmissions made by the node is lower than the number of linearly independent packets 
received, the node tries to access the channel with probability $p_{a}=1$. If the node has a number of stored packets which is lower than or equal to the number of those transmitted, instead, it tries to access the channel with probability $p_{a}=\zeta-1$.

When a node receives a packet from another node, it checks whether the packet is linearly independent with the stored packets and, if this is the case, the new packet is stored. If the received packet is not linearly independent with the stored ones, it is discarded.

We recall that this is only one possible cooperative scheme which is not necessarily the optimal one. For instance, different mechanisms for medium access and transmit packet selection can be adopted.

\section{Implementation Aspects}

According to the DVB-SH standard we consider a source symbol size of 1024 bytes each. At the terminal nodes each source symbol is divided into $n_{s s}$ subsymbols, each of which containing $\frac{1024}{n_{s s}}$ bytes. Each of these subsymbols is multiplied by a randomly chosen coefficient in a field with $q=\frac{1024}{n_{s s}}=2^{n}$ elements. The coefficient is the same for all subsymbols within a symbol. In this way the complexity of the network encoder/decoder can be kept at a reasonable level [12]. A field size of $2^{8}$ or $2^{16}$ (one or two bytes) may constitute a valid choice. The NC is applied as in [12], adding the encoding vector at the end of each packet. Thus, for a $K$ symbols generation, a header with $K \times q$ bits is appended to each symbol. The loss in spectral efficiency is then $(K q) / 8192$. Assuming coefficients of 1 byte are used, the loss becomes $K / 1024$. In order to keep the loss at a reasonable value we should limit the size of the generation. For instance, if generations of $K=100$ symbols are used, the loss is below $10 \%$. The adoption of small generation sizes has the drawback that the code efficiency is reduced. For example, it is known that the efficiency of the Raptor code increases with the source block. A tradeoff is to be found between the size of the coefficients (that influences the efficiency in the information distribution among the nodes) and the generation size (which influences the performance of Raptor code). Apart from such tradeoff, we point out that there is a further advantage in using a relatively short generation size. As a matter of facts, since the short interleaver is always used together with IFEC protection, a block of small size would make the data readily available to the upper layer sooner than in the case of large blocks, thus reducing the decoding delay. In Section VII we show the gap between the asymptotic results obtained in Section IV and the simulation results 
obtained in the same setup but with the 3GPP Raptor code, having finite block-length.

\section{NUMERICAL RESUlTS}

Fig. 4 shows the coverage $\Omega$, obtained evaluating numerically Eqn. (20), plotted against the rate at physical level $r$ for a fixed message rate $R$ and different network sizes. The relative lower bounds and the coverage curve in case of no cooperation are also shown. In the simulation we set $R=2 / 3, p_{a}=0.2, \Gamma_{N}=10 \mathrm{~dB}$ in the N-N channel, $\mu=3$ and $\sigma=1$ in the S-N channel. It is interesting to note how, for the considered network sizes, increasing the number of nodes also increases the achievable rate $r$ for a given $\Omega$. In other words, the higher the number of nodes, the higher the probability that all the information broadcasted by $S$ reaches the network, i.e., is received by at least one node. Once the information has reached the network, it can be efficiently distributed among the terminals through random linear network coding. An important gain in the transmission rate can be observed, with an increase of about $0.4 \mathrm{bit} / \mathrm{s} / \mathrm{Hz}$ when passing from no cooperation to cooperation in a network with 2 nodes, and about $1 \mathrm{bit} / \mathrm{s} / \mathrm{Hz}$ in case of a network with 4 nodes. An important point is that this result is achieved without any feedback to the source or any packet request among nodes, as the decision on whether to encode and transmit or not is taken autonomously by each terminal depending on the probability of media contention $p_{a}$. The lower bound is fairly tight for $M=2$ and $M=4$.

In Fig. 5 the coverage is plotted against the probability of transmission attempt $p_{a}$ (fixed for each node) for $M=4, \Gamma_{N}=10 \mathrm{~dB}, r=1 \mathrm{bit} / \mathrm{s} / \mathrm{Hz}$ and $R=2 / 3$. It is interesting to note that relatively small values of $p_{a}$ (lower than 0.15 for the asymptotic case) are sufficient to achieve full coverage for values of $r$ and $R$ which are of practical interest. We further observe that the lower bound tightly approximates the simulated theoretical curve. The coverage for the non cooperative case in the setup considered in Fig. 5 is 0, coherently with Fig. 4.

\section{CONCLUSIONS}

In this chapter we investigated the possibility of using a cooperative approach for providing missing coverage in heterogeneous LMS networks. We carried out an analytical study considering a mathematically tractable and yet practically interesting network model, in which fading and shadowing effects in the communication channels as well as the medium access mechanism of the cooperating nodes have been taken into account. By applying the Max-flow Min-cut theorem 


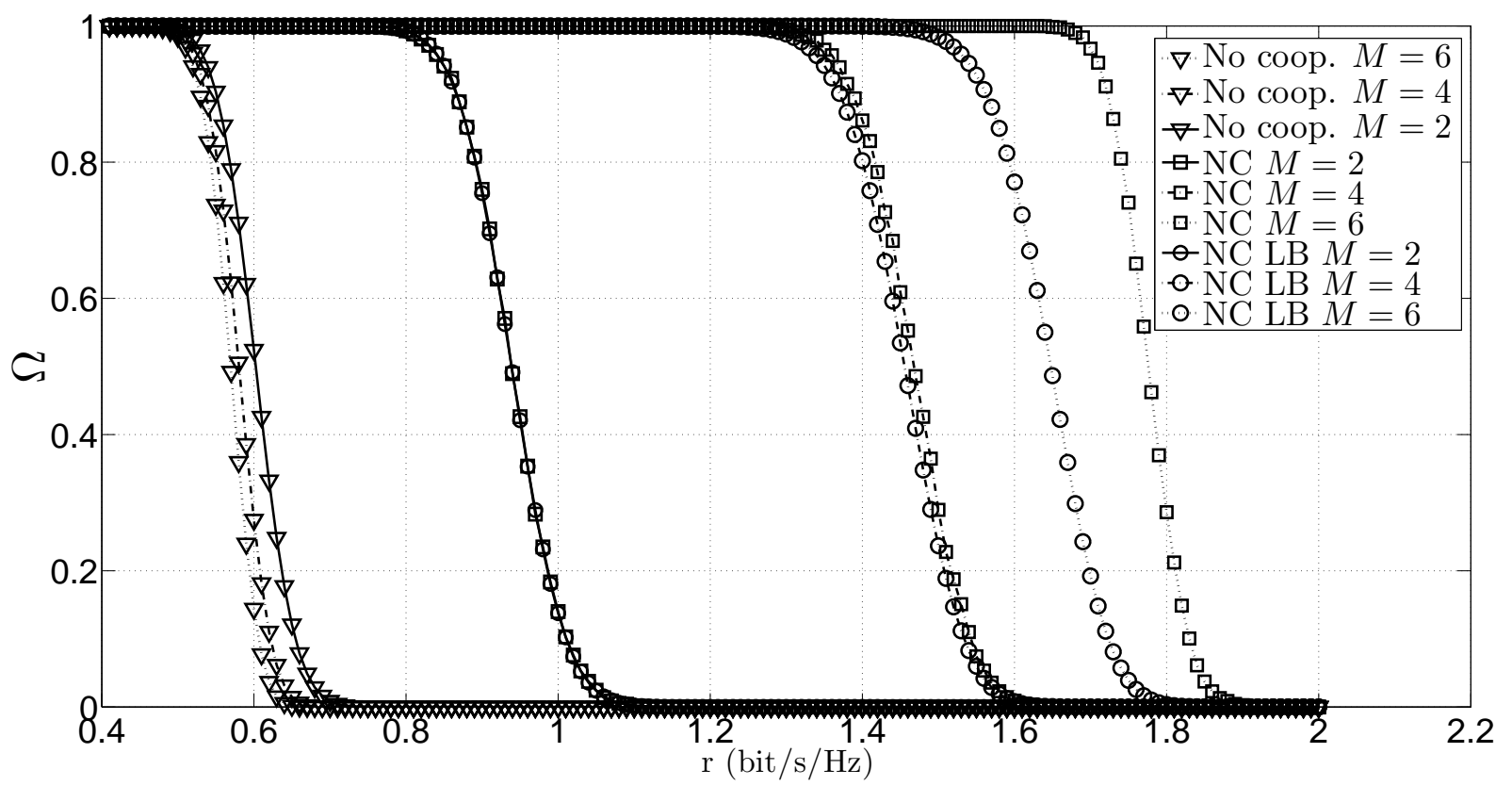

Fig. 4. Coverage $\Omega$ plotted against rate at physical layer $r$ in the cooperative case for different values of $M$. The lower bound and the non cooperative case are also shown. In the simulation we set $R=2 / 3$ messages/slot, $p_{a}=0.2, \Gamma_{N}=10 \mathrm{~dB}$ in the $\mathrm{N}-\mathrm{N}$ channels, $\mu=3$ and $\sigma=1$ in the S-N channel.

we derived an analytical lower bound on the coverage as a function of both the information rate at physical layer and the rate of innovative packets injected in the network per unit-time. Our results show a tradeoff between the coverage and the rate at which the information can be injected in the network, and at the same time quantify the gain derived from node cooperation. We showed that, at least for the considered network sizes, the gain grows with the number of terminals, contrary to what happens in the non cooperative case.

Based on the considered theoretical model we suggested a practical cooperative scheme which leverages on network coding for enhancing coverage in heterogeneous vehicular LMS systems adopting DVB-SH in the satellite segment. 


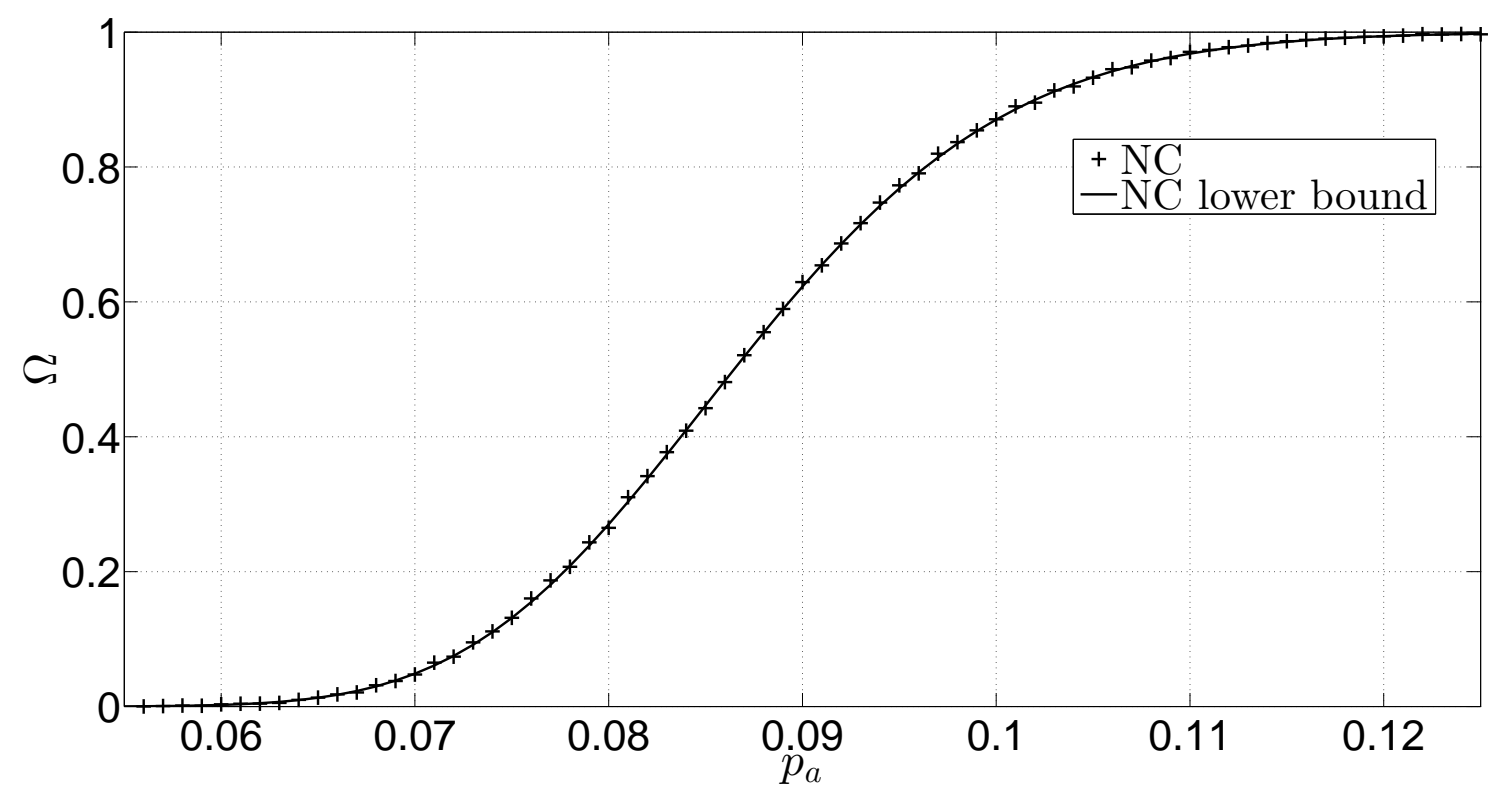

Fig. 5. Coverage $\Omega$ plotted against the probability of media contention $p_{a}$ in the cooperative case for a network with $M=4$ and $\Gamma_{N}=10 \mathrm{~dB}$. The lower bound $\Omega_{L B}$ is also shown. In the simulation we set $R=2 / 3$ messages $/ \mathrm{slot}, r=1 \mathrm{bit} / \mathrm{s} / \mathrm{Hz}$, $\mu=3$ and $\sigma=1$ in the $\mathrm{S}-\mathrm{N}$ channel.

\section{REFERENCES}

[1] Exalted Project, "First report on LTE-M algorithms and procedures," http://www.ict-exalted.eu, Aug. 2011.

[2] European Telecommunications Standards Institute, "ETSI TS 102584 V1.2.1, Digital Video Broadcasting (DVB); DVB-SH Implementation Guidelines Issue 2.” Jan. 2011.

[3] Inmarsat, "Broadband Global Area Network (BGAN)," http://www.inmarsat.com/services/bgan.

[4] T. M. Cover and J. A. Thomas, Elements of Information Theory, second edition ed. John Wiley \& Sons, 2006.

[5] D. S. Lun, M. Médard, R. Koetter, and M. Effros, "On coding for reliable communication over packet networks," Physical Comm., vol. 1, no. 1, pp. 3-20, 2008. [Online]. Available: http://www.sciencedirect.com/science/article/pii/S1874490708000086

[6] G. Cocco, C. Ibars, and O. D. R. Herrero, "Cooperative satellite to land mobile gap-filler-less interactive system architecture," in IEEE Advanced Satellite Mobile Systems Conf., Cagliari, Italy, Sep. 2010.

[7] Y. Tseng, S. Ni, Y. Chen, and J. Sheu, "The broadcast storm problem in a mobile ad hoc network," Wireless Networks, vol. 8, pp. 153-167, 2002.

[8] J. Wu and F. Dai, "A generic distributed broadcast scheme in ad hoc wireless networks," IEEE Trans. on Computers, vol. 53, no. 10, pp. 1343-1354, Oct. 2004.

[9] A. Vanelli-Coralli, G. E. Corazza, G. K. Karagiannidis, P. T. Mathiopoulos, D. S. Michalopoulos, C. Mosquera, S. Papaharalabos, and S. Scalise, "Satellite communications: Research trends and open issues," in Int'l Workshop on Satellite and Space Comm., Toulouse, France, Sept. 2007. 
[10] S. Morosi, E. D. Re, S. Jayousi, and R. Suffritti, "Hybrid satellite/terrestrial cooperative relaying strategies for DVB-SH based communication systems," in European Wireless Conf., Aalborg (Denmark), May 2009.

[11] T. Ho, M. Médard, R. Koetter, D. R. Karger, M. Effros, J. Shi, and B. Leong, "A random linear network coding approach to multicast," IEEE Trans. on Info. Theory, vol. 52, no. 10, pp. 4413-4430, Oct. 2006.

[12] P. A. Chou, Y. Wu, and K. Jain, "Practical network coding," in IEEE Allerton Conf. on Communication, Control, and Computing, Urbana-Champaign, IL, U.S.A., Oct. 2003.

[13] R. Ahlswede, C. Ning, S.-Y. R. Li, and R. W. Yeung, "Network information flow," IEEE Trans. on Info. Theory, vol. 46, no. 4, pp. 1204-1216, July 2000.

[14] M. Sardari, F. Hendessi, and F. Fekri, "Infocast: A new paradigm for collaborative content distribution from roadside units to vehicular networks," in Annual IEEE Comm. Society Conf. on Sensor, Mesh and Ad Hoc Comm. and Networks, Rome, Italy, June 2009

[15] P. Cataldi, A. Tomatis, G. Grilli, and M. Gerla, "A novel data dissemination method for vehicular networks with rateless codes," in IEEE Wireless Comm. and Networking Conf. (WCNC), Budapest, Hungary, Apr. 2009.

[16] ANSI/IEEE Std 802.11, 1999 Edition (R2003), Institute of Electrical and Electronics Engineers (IEEE), http://ieeexplore.ieee.org/xpl/mostRecentIssue.jsp?punumber=9543, 1999.

[17] S. Deb, M. Effros, T. Ho, D. R. Karger, R. Koetter, D. S. Lun, M. Mdard, and N. Ratnakar, "Network coding for wireless applications: A brief tutorial," in IEEE Int'l Workshop on Wireless Ad-hoc Networks, London, UK, May 2005.

[18] H. Suzuki, "A statistical model for urban radio propogation," IEEE Trans. on Comm., vol. 25, no. 7, pp. 673-680, July 1977.

[19] E. Lutz, D. Cygan, M. Dippold, F. Dolainsky, and W. Papke, "The land mobile satellite communication channel-recording, statistics, and channel model," IEEE Trans. on Vehicular Technology, vol. 40, no. 2, pp. 375-386, May 1991.

[20] G. Cocco, C. Ibars, and N. Alagha, "Cooperative coverage extension in heterogeneous Machine-to-Machine networks," in Globecom 2012 Workshop: Second International Workshop on Machine-to-Machine Communications 'Key' to the Future Internet of Things, Anaheim, CA, U.S.A., Dec. 2012.

[21] "Digital Video Broadcasting (DVB); Upper Layer Forward Error Correction in DVB. DVB Document A148," http://www.dvb.org/, Mar. 2010.

[22] European Telecommunications Standards Institute, "ETSI TS 102472 V1.1.1, Digital Video Broadcasting (DVB); IP Datacast over DVB-H: Content Delivery Protocols,” June 2006.

[23] — _DVB-SH implementation guidelines, DVB BlueBook A120," http://www.dvb-h.org/, May 2008.

[24] G. Cocco, N. Alagha, and C. Ibars, "Network-coded cooperative extension of link level FEC in DVB-SH," in AIAA International Communications Satellite Systems Conf., Nara, Japan, Dec. 2011. 